\title{
New measurement of orthopositronium decay rate
}

\author{
S.Asai* ${ }^{*}$ S.Orito, and N.Shinohara ${ }^{\dagger}$ \\ Department of Physics, Faculty of Science, \\ University of Tokyo, Tokyo 113, Japan
}

(Submitted to Physics Letters B) Sw9532

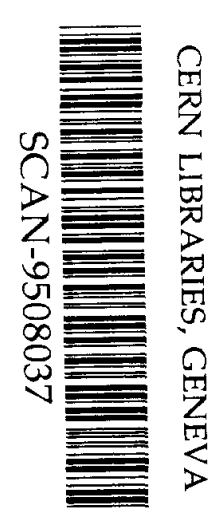

\section{ICEPP}

International Center for Elementary Particle Physics, University of Tokyo 7-3-1 Hongo, Bunkyo-ku, Tokyo 113, Japan

\footnotetext{
"E-mail address: asai@cernvm.cern.ch

†Institute of Physics, College of Arts and Sciences, University of Tokyo, 3-8-1 Komaba, Meguro, Tokyo 153, Japan
} 
UT-ICEPP 95-08

June 1995

\title{
New measurement of orthopositronium decay rate
}

\author{
S.Asai*, S.Orito, and N.Shinohara ${ }^{\dagger}$ \\ Department of Physics, Faculty of Science, \\ University of Tokyo, Tokyo 113, Japan
}

\begin{abstract}
The orthopositronium decay rate is measured with an entirely new method, in which the pickoff annihilation rate is directly measured as a function of time with a high-resolution germanium detector. The decay rate can be determined without the ambiguities of the thermalization nor of the extrapolation, which might have been sources of the main systematic errors in all the previous experiments. Two independent measurements are carried out with two different types of $\mathrm{SiO}_{2}$ powder, resulting in decay rate values consistent with each other. The combined result is $\lambda_{0}=7.0398$ \pm 0.0025 (stat.) \pm 0.0015 (sys.) $\mu \mathrm{s}^{-1}$, which is consistent with the QED prediction, and differs by $2.9-4.1 \sigma$ from three recent measurements.
\end{abstract}

(Submitted to Physics Letters B)

\section{ICEPP}

International Center for Elementary Particle Physics, University of Tokyo

7-3-1 Hongo, Bunkyo-ku, Tokyo 113, Japan

\footnotetext{
*E-mail address: asai@cernvm.cern.ch

IInstitute of Physics, College of Arts and Sciences, University of Tokyo, 3-8-1 Komaba, Meguro, Tokyo 153, Japan
} 


\section{Introduction}

Positronium (Ps), the bound state of an electron and a positron, is a purely leptonic system, and the triplet $\left(1^{3} S_{1}\right)$ state of Ps, orthopositronium(o-Ps), decays slowly into three photons. Precise measurement of this decay rate gives us direct information about quantum electrodynamics(QED), since to higher order the constituent leptons interact only through the electromagnetic interactions. Three precision measurements $[1,2,3]$ of the 0 - Ps decay rate were recently performed, which reported decay rate values much larger (by 1400, 1300 and 920 ppm) than the theoretical QED calculation corrected up to $O\left(\alpha^{2}\right)[4,5,6]$. This discrepancy, being $6.7,6.3$ and $4.3 \sigma$, is statistically very significant. In an effort to clarify the discrepancy, various exotic decay modes have been searched for, without any evidence so far $[7,8,9,10,11,12]$.

Because some portion of o-Ps inevitably causes the 'pickoff' annihilation by colliding with the atomic electrons of the target material, the observed o-Ps decay rate, $\lambda_{o b s}$, is the sum of the the intrinsic decay rate, $\lambda_{0}$, and the pickoff annihilation rate, $\lambda_{\text {pickof } f}$, i.e.

$$
\lambda_{\text {obs }}(t)=\lambda_{0}+\lambda_{\text {pickoff }}(t) .
$$

The $\lambda_{\text {pickoff }}$ is proportional to the rate at which the o-Ps collides with the materials, and is consequently proportional to the densities of the target materials and to the velocity of the o-Ps. Since the o-Ps has a kinetic energy of about 1 $\mathrm{eV}$ immediately after formation, and slows down by elastic collisions toward the ultimate thermal energy of about $0.03 \mathrm{eV}$ ('thermalization'), the corresponding pickoff annihilation rate is a function of time, which depends on the properties of the material. In all the previous experiments, the $\lambda_{o b s}$ 's were measured by varying densities of the target materials, and the extrapolation to zero densities yielded the $\lambda_{0}$, assuming that the velocity of the $o-P s$ was constant. However, there seems to exist no concrete evidence to support this assumption, and the unthermalized o-Ps could result to a large systematic error $[13,14]$. Furthermore the assumption of the linear dependence of $\lambda_{\text {pickoff }}$ on the densities of the target materials is only valid, if the collision between the o-Ps and the material is like the one between single rigid balls. However the collisions of the o-Ps with the materials are conjectured to be more complicated due to the processes such as the multiple scattering at the surface of the materials [15]. Therefore the linear extrapolation to zero density might be a source of another systematic error.

We report here on a new measurement of the o-Ps decay rate with the following entirely new method, which is free from above-mentioned systematic errors. From Eq.(1), the population of o-Ps, $N(t)$, is expected to obey the following equation:

$$
N(t)=N_{0} \exp \left(-\lambda_{0} \int_{0}^{t}\left(1+\frac{\lambda_{\text {pickoff }}\left(t^{\prime}\right)}{\lambda_{0}}\right) d t^{\prime}\right)
$$


Because the final state of the pickoff annihilation is 2-body, the emitted photon is monochromatic at $511 \mathrm{keV}$, while the energy distribution of the photon from the $3 \gamma$-decay is continuous. Hence the ratio, $\lambda_{\text {pickof } f}(t) / \lambda_{0}$, can be determined from the energy spectrum of the emitted photon, if the energy and the timing information are simultaneously recorded. Since the determined ratio includes all the information about the $0-$ Ps thermalization, the $\lambda_{0}$ can be obtained by fitting the observed time spectrum with the Eq.(2), without any ambiguity of the thermalization. Furthermore the $\lambda_{0}$ is directly obtained in this method, and has no ambiguity of the extrapolation mentioned above.

\section{$2 \quad$ Experimental setup}

Figure 1 shows a diagram of the experimental setup [13]. A ${ }^{22} \mathrm{Na}$ positron source of $0.02 \mu \mathrm{Ci}$ with a $2 \mathrm{~mm}$ spot diameter is sandwiched between two sheets of plastic scintillator (NE104), each with a diameter of $12 \mathrm{~mm}$ and thickness 100 $\mu \mathrm{m}$. The source is held by a cone made of aluminized mylar of $25 \mu \mathrm{m}$ thickness, and is placed at the centre of a vacuum container of diameter $48 \mathrm{~mm}$ made of 500 $\mu \mathrm{m}$ thick glass. The container is filled with silica powder $\left(\mathrm{SiO}_{2}\right)$ of density 0.03 $\mathrm{g} / \mathrm{cm}^{3}$ and grain size $7 \mathrm{~nm}$ in diameter, and is evacuated to $10^{-2}$ torr. Most of the emitted positrons pass through the scintillator, transmitting a light pulse to a photomultiplier(Trigger PMT: Hamamatsu H-3165-04) and forming Ps when stopped in the silica powder.

The energy and timing information of the photon emitted from the decay of o-Ps are measured with a germanium semiconductor detector(diameter: $61 \mathrm{~mm}$, thickness: $69 \mathrm{~mm}$, Ortec GEM 38195) and with four CsI(Tl) scintillators(each crystal size: $56 \times 56 \times 56 \mathrm{~mm})$. The main purpose of the germanium detector is the determination of the $\lambda_{\text {pickof } f}(t) / \lambda_{0}$ by the shape of the energy spectrum. The time spectrum can be obtained, in principle, both from the germanium detector and from the CsI scintillators. In practice, the time spectrum from the CsI scintillators is used to be fitted with Eq.(2), because they have efficiencies much higher than the one of the germanium detector. The energy resolutions and absolute peak efficiencies of these detectors are determined as a function of the photon-energy using the line- $\gamma$ from ${ }^{57} \mathrm{Co},{ }^{152} \mathrm{Eu},{ }^{85} \mathrm{Sr},{ }^{137} \mathrm{Cs}$, and ${ }^{22} \mathrm{Na}$. The energy resolutions of the germanium detector (CsI scintillator) are $1.3(42)$ and 1.8 (69) keV FWHM at 511 and $1275 \mathrm{keV}$, respectively. During the measurement, a lead sheet of $2.08 \mathrm{~mm}$ thickness is placed in front of the germanium detector in order to eliminate the contribution of two low-energy photons from a $3 \gamma$-decay of o-Ps simultaneously hitting the germanium detector.

The trigger and the CAMAC data-acquisition system are described as follows. The output from the trigger PMT is put into a discriminator whose output provides both the start signal to the time-to-digital converters (TDC) (Hohshin 
C006 and REPIC RPC-061) and the trigger signal to the CAMAC system. Two different types of TDCs are used in parallel to check the data with each other. Both TDCs are calibrated every two months by the time-calibrator (Ortec 462) with an intrinsic time accuracy of $50 \mathrm{ppm}$, and show to be stable within $60 \mathrm{ppm}$ during the measurement. One output from the preamplifier of the germanium detector is fed through a fast-filter amplifier (Ortec 579) into a constant-fraction discriminator (Ortec 583) whose output is used as the stop signal for the TDCs. The differential and integral time of the fast-filter amplifier are optimized in order to obtain a good time resolution of $6 \mathrm{~ns}$ rms. The other preamplifier output is amplified by a spectroscopy amplifier (Ortec 673) whose outputs are fed into a peak-holding amplitude-to-digital converter (ADC) (Hohshin C011) and charge-sensitive ADC (REPIC RPC-022, hereafter denoted as 'BASE-ADC') to record the energy information and the base-line condition prior to the event, respectively.

One output from each CsI scintillator is fed into a discriminator (Lecroy 825) whose output is used as the stop signal for the TDCs, resulting to the time resolution of $4.8 \mathrm{~ns}$ rms. Another two outputs of the scintillator are also provided into a charge-sensitive ADC (Lecroy 2249W) and the BASE-ADC in order to record energy information and the base-line condition, respectively. The electronics described above is provided individually to four CsI scintillators.

The data for the decay rate measurement are collected from 25 runs for a total live time of $1.30 \times 10^{7} \mathrm{~s}$, and a total of $4.64 \times 10^{9} \beta^{+}$emissions are observed. During the measurement, the room temperature is controlled within $\pm 0.3^{\circ} \mathrm{C}$ to ensure stability of the amplifiers, the ADCs, and the TDCs. The narrow width (1.8 keV FWHM) of the observed accidental $1275-\mathrm{keV}$ line- $\gamma$ peak indicates that excellent energy resolution and stability are obtained throughout the data-taking period.

\section{Analysis and discussion}

At first, the $\lambda_{\text {pickof } f}(t) / \lambda_{0}$ is determined by using the energy spectrum measured with the germanium detector. The energy spectrum of the photon emitted from a pure sample o-Ps (called here 'o-Ps spectrum') is obtained by subtracting the accidental contribution from the spectrum at the time window of 160-710 ns. Fig. 2 (a) shows the resultant o-Ps spectrum, in which the peak at $511 \mathrm{keV}$ due to the pickoff annihilation is clearly visible on top of the continuous spectrum of the $3 \gamma$-decay.

The expected energy spectrum of the $3 \gamma$-decay, which is denoted as ' $3 \gamma$ spectrum', is calculated by the Monte Carlo simulation, in which the setup geometry and various material distributions are reproduced in detail. For every simulated 
event, three photons are generated according to an order- $\alpha$-corrected energy spectrum [16]. Successive photoelectric, Compton, or Rayleigh scattering interactions of every photon are then followed through the materials until all the energy is dissipated. The response function of the detector is determined based on the measured spectra of monochromatic $\gamma$ rays from ${ }^{137} \mathrm{Cs}$ and ${ }^{85} \mathrm{Sr}$ as well as on the annihilation line from ${ }^{22} \mathrm{Na}$, and is folded in the simulation.

As seen in Fig. 2 (a), the $3 \gamma$ spectrum reproduces well the o-Ps spectrum over a wide energy region below $508 \mathrm{keV}$. Although the simulation is elaborated, the resultant $3 \gamma$ spectrum is very insensitive to the details of the simulation, and has little ambiguity, because the sharp falloff of the spectrum at $511 \mathrm{keV}$ is purely due to the phase-space cutoff and is almost entirely determined by the absolute energy scale and by the energy response function of the germanium detector, which have been accurately determined by measurements. One potential problem could have been the contribution of events in which two low-energy photons from a $3 \gamma$ decay simultaneously hit the detector. Although such events are taken into account in the simulation, their occurrence would cause the energy spectrum to abruptly increase above $511 \mathrm{keV}$, and be more sensitive to systematic errors of the simulation. However, as seen in the lower hatched histogram in this figure, such events are reduced to a negligible level by the lead shield placed in front of the detector.

Figure 2 (b) shows an enlarged view of the o-Ps spectrum after subtracting the $3 \gamma$ spectrum. The peak observed in this spectrum (hereafter denoted as the 'peak spectrum') can be well fitted with the measured response function of the germanium detector. Furthermore, the fitted centre of the peak is located exactly at $511 \mathrm{keV}(511.00 \pm 0.02 \mathrm{keV})$, showing that the $3 \gamma$ subtraction is performed properly. Thus this peak spectrum can be considered to be a pure sample of the pickoff annihilation, and the pickoff annihilation occuring in this time window can be counted from the detected number under this peak. The number of $o-P s$ decaying into $3 \gamma$ 's is also counted from the o-Ps spectrum, and the $\lambda_{\text {pickof } f} / \lambda_{0}$ can be simply calculated. The resultant $\lambda_{\text {pickof } f} / \lambda_{0}$ is stable against various systematic errors; e.g., it depends only on the relative efficiency of the germanium detector and not on its absolute efficiency.

These calculations of the $\lambda_{\text {pickoff }} / \lambda_{0}$ are performed for various time windows, and its time dependence is shown in Fig. 2(c). Because the fractional energy loss of $\mathrm{o}-\mathrm{Ps}$ per collision is dependent on its energy in $\mathrm{SiO}_{2}$ powder, the time dependence of the average kinetic energy of $0-$ Ps at time $t, \overline{E(t)}$, can be described as $[15,17]$ :

$$
\frac{d}{d t} \overline{E(t)}=-\sqrt{2 m_{p s} \overline{E(t)}}\left(\overline{E(t)}-\frac{3}{2} k_{B} T\right)\left(\frac{2}{\bar{M} \bar{L}}\right)\left(\overline{\frac{E(t)}{k_{B} T}}\right)^{\kappa},
$$

where $m_{p s}, \bar{M}$, and $\bar{L}$ are the mass of $\mathrm{o}-\mathrm{Ps}$, the effective mass at the surface of the $\mathrm{SiO}_{2}$ grain, and the mean distance between the grains. Since the 
pickoff annihilation rate is proportional to the average velocity of $o-P s$, the $\lambda_{\text {pickoff } f}(t) / \lambda_{0}(\equiv \theta(t))$ is expected to obey the following differential equation:

$$
\frac{d}{d t} \theta(t)=-C\left(\theta(t)^{2}-\theta_{\infty}^{2}\right) \theta(t)^{2 \kappa}
$$

where $\theta_{\infty} \equiv \theta(t=\infty)$ and $C$ are the constants. The measured $\lambda_{\text {pickoff }} / \lambda_{0}$ 's are fitted with this equation, and the fitting result is superimposed in this figure. As indicated in this figure, the observed data are well fitted, and the o-Ps in the $\mathrm{SiO}_{2}$ powder takes about $600 \mathrm{~ns}$ to be well thermalized.

Data from the CsI scintillators are collected in parallel with the germanium detector. Figure 3 shows the time spectrum from these scintillators at the photonenergy window of 300-410 keV, in which a sharp peak of the prompt annihilation is followed by the exponential decay of o-Ps and subsequently by the constant accidentals. The o-Ps decay curve has been observed in a wide region of about $1.2 \mu$ s corresponding to eight times the o - Ps lifetime, because of the weak positron source of $0.02 \mu \mathrm{Ci}$ used in this experiment.

The time spectrum is fitted by the method of least squares with the following function [13]:

$$
F(t)=\exp \left(-R_{\text {stop }} t\right)\left[\left(1+\frac{\epsilon_{\text {pickoff }}}{\epsilon_{3 \gamma}} \frac{\lambda_{\text {pickoff }}(t)}{\lambda_{0}}\right) N(t)+C\right],
$$

where $N(t)$ is already given in Eq. (2). In this function, $\epsilon_{\text {pickoff }}$ and $\epsilon_{3 \gamma}$ are defined as the probabilities that photons emitted from the pickoff annihilation and the $3 \gamma$-decay of o-Ps deposit the energy of $300-410 \mathrm{keV}$ on the CsI scintillator, respectively. The ratio of $\epsilon_{\text {pickoff }} / \epsilon_{3 \gamma}$ is calculated to be 0.27 by using the Monte Carlo simulation. Because the effect of $\frac{\epsilon_{\text {pickoff }} \frac{\lambda_{\text {pickoff }}(t)}{\epsilon_{3 \gamma}}}{\lambda_{0}}$ on the decay rate is estimated to be only $110 \mathrm{ppm}$, the obtained decay rate does not depend much on the simulation. The $R_{\text {stop }}$ in this function is the measured stop rate of the CsI scintillator. Three remaining free parameters of fitting are then $\lambda_{0}, N_{0}$, and $C$.

As seen in the fitting curve shown in Fig. 3 (almost indistinguishable from the data), we have an excellent fitting over the entire time span with $\chi^{2}$ of 223.1 / 210 points. The fitted $\lambda_{0}$ is $7.0403 \pm 0.0035$ (stat.) $\mu \mathrm{s}^{-1}$, which includes also the statistic error propagated from the determination of the $\lambda_{\text {pickof } f}(t) / \lambda_{0}$. This result is checked to be stable against various systematics, e.g., the time span for the fitting, the selected energy window, and the characters of the TDCs. The consistent result $\left(7.038 \pm 0.009 \mu \mathrm{s}^{-1}\right)$ is also obtained by fitting the time spectrum from the germanium detector.

Systematic errors on this measurement are summarized [13] in Table 1 . The total systematic error is calculated as a quadratic sum of the individual errors because they are independent from each other. It is important to note that 
the error propagated from the $\lambda_{\text {pickof } f}(t) / \lambda_{0}$ to final result is small, since the $\lambda_{\text {pickoff }}(t) / \lambda_{0}$ are small as shown in Fig. 2(c). The systematic error of the Monte Carlo simulation is estimated by comparing the peak spectrum with the measured response function. Because the simulated data affect mainly the lower side of the peak spectrum [18], the shape of the peak spectrum, especially an asymmetry around the peak centre [19], is very sensitive to the reliability of the Monte Carlo simulation. The obtained peak spectrum is checked to be consistent with the response function by varying the simulated data forcibly, from which the systematic error of the simulation is estimated.

An independent measurement is carried out with a different $\mathrm{SiO}_{2}$ powder of density $0.05 \mathrm{~g} / \mathrm{cm}^{3}$ and grain size $14 \mathrm{~nm}$ in order to check that the resultant decay rate does not depend on the characteristics of the powder. Although the observed $\lambda_{\text {pickof } f}(t) / \lambda_{0}$ differs very much from the first powder, as expected from the strong dependence of the $\lambda_{\text {pickof } f}(t) / \lambda_{0}$ on the characters of the powder, we obtain decay rate $\left(7.0393 \pm 0.0035\right.$ (stat.) $\left.\mu \mathrm{s}^{-1}\right)$ consistent with the value obtained with the first powder.

\section{Conclusion}

The measurements of the o - Ps decay rate are carried out with an entirely new method, in which the pickoff annihilation rate is directly measured as a function of time. The decay rate is determined without the ambiguity of the thermalization nor of the extrapolation, which, we suspect, were the sources of the main systematic errors in all the previous experiments. Two measurements with different types of $\mathrm{SiO}_{2}$ powder are carried out independently, the results being consistent to each other. Combining these two results, we obtain $\lambda_{0}=$ $7.0398 \pm 0.0025$ (stat.) \pm 0.0015 (sys.) $\mu \mathrm{s}^{-1}$. As shown in Fig. 4, this result is consistent with the QED prediction $\left(7.0420 \mu \mathrm{s}^{-1}\right)[4,5,6]$, and differs by 4.1 and $4.0 \sigma$ from the two recent experiments using gases $[1,2]$, and by $2.9 \sigma$ from the recent cavity experiment [3].

We wish to thank Prof. T. Hyodo (Univ. of Tokyo) and Dr. Y. Nagashima (Univ. of Tokyo) for very useful suggestions and discussions especially about the o- Ps thermalization. It is a pleasure to thank Prof. G.S. Adkins (Franklin \& Marshall College) for calculating the $O(\alpha)$ corrected energy spectrum. Sincere gratitude is expressed to Mr. Y. Nakamura (Japan Radioisotope Association) for the preparation of the positron source, and Mr. A. Kohinata and Mr. Uchida (Nippon Aerosil Ltd.) for the supply of the special silica powders and for the discussions about the characteristic of these powders. 


\section{References}

[1] C.I. Westbrook et al., Phys. Rev. Lett. 58 (1987) 1328: C.I. Westbrook, Ph. D thesis, Univ. of Michigan 1987.

[2] C.I. Westbrook, D.W. Gidley, R.S. Conti and A. Rich, Phys. Rev. A 40 (1989) 5489.

[3] J.S. Nico, D.W. Gidley, A. Rich and P.W. Zitzewitz, Phys. Rev. Lett. 65 (1990) 1344.

[4] W.E. Caswell, G.P. Lepage and J. Sapirstein, Phys. Rev. Lett. 38 (1977) 488; G.S. Adkins, Ann. Phys. (NY) 146 (1983) 78; G.S. Adkins, A.A. Salahuddin and K.E. Schalm, Phys. Rev. A 45 (1992), ibid. Phys. Rev. A 45 (1992) 7774.

[5] A.P. Burichenko, Phys. At. Nucl. 56 (1993) 640; P.L. Labelle and P. Lepage, Phys. Rev. Lett. 72 (1994) 2006; E.A. Kuraev, T.V. Kukhto, and Z.K. Silagadze, Sov. J. Nucl. Phys. 51 (1990) 1036; A.I. Milstein and I.B. Khriplovich, JETP 79 (1994) 379; S.G. Karshenboim, Phys. At. Nucl. 56 (1993) 1710.

[6] W.E. Caswell and G.P. Lepage, Phys. Rev. A 20 (1979) 36; I.B. Khriplovich and A.S. Yelkhovsky, Phys. Lett. B 246 (1990) 520; S.G. Karshenboim, JETP 76 (1993) 541.

[7] S. Asai, S. Orito, K. Yoshimura and T. Haga, Phys. Rev. Lett. 66 (1991) 2440; S. Orito, K. Yoshimura, T. Haga and M. Tsuchiaki, Phys. Rev. Lett. 63 (1989) 597.

[8] T. Maeno, M. Fujikawa, J. Kataoka, Y. Nishihara, S. Orito, K. Shigekuni and Y. Watanabe, Phys. Lett. B 351 (1995) 574; S. Asai, K. Shigekuni, T. Sanuki, and S. Orito, Phys. Lett. B 323 (1994) 90; M. Tsuchiaki, S. Orito, T. Yoshida, and M. Minowa, Phys. Lett. B 236 (1990) 81.

[9] T. Mitsui, K. Maki, N. Muramoto, T. Sato and S. Asai, ICEPP Report UT-ICEPP 93-14 (1993).

[10] T. Mitsui, R. Fujimoto, Y. Ishisaki, Y .Ueda, Y. Yamazaki, S. Asai and S. Orito, Phys. Rev. Lett. 70 (1993) 2265.

[11] S. Asai, S. Orito, T. Sanuki, M. Yasuda and T. Yokoi, Phys. Rev. Lett. 66 (1991) 1298; D.W. Gidley, J.S. Nico and M. Skalsey, Phys. Rev. Lett. 66, 1302 (1991); A.P. Mills, Jr., and D.M. Zuckerman, Phys. Rev. Lett. 64 (1990) 2637.

[12] K. Marko and A. Rich, Phys. Rev. Lett. 33 (1974) 980.

[13] S. Asai, Doctoral thesis, Univ. of Tokyo, ICEPP Report UT-ICEPP 94-08 (1994). 
[14] S. Asai, T. Hyodo, Y. Nagashima, T. Chang and S. Orito, Materials Science Forum, 175-178 (1995) 619.

[15] T. Hyodo and Y. Nagashima (private communication).

[16] G.S. Adkins (private communication).

[17] T. Hyodo, M. Kakimoto, T.B. Chang, J.Deng, T. Akahane, T. Chiba, B.T.A Mckee, and A.T. Stewart, in Positron Annihilation, edited by L.DorikensVanbraet, M. Dorikens and D. Segers (World Scientific, Singapore, 1989), p.878.

[18] The sharp falloff of the simulated spectrum at $511 \mathrm{keV}$ is only smeared by the germanium detector's resolution (FWHM $=1.3 \mathrm{keV})$, but the peak spectrum has the large width of FWHM $=2.6 \mathrm{keV}$ due to the Fermi momentum of the atomic electrons.

[19] M.A. Hammed, P.W. Gray, A.H. Naboulsi, and T.D. MacMahon, Nucl. Inst. Methods A334 (1993) 543. 


\begin{tabular}{|l|r|}
\hline Source & Error (ppm) \\
\hline \hline Accuracy of the time calibrator & \pm 50 \\
\hline Stability of TDC & \pm 60 \\
\hline Relative efficiency of the Ge detector & -80 and +50 \\
\hline Monte Carlo simulation of the $3 \gamma$ decay & -150 and +190 \\
\hline Calculation of $\lambda_{\text {pickoff }} / \lambda_{0}$ & \pm 50 \\
\hline Dependence on the BASE-ADC selection & -70 \\
\hline Energy correction of the CsI scintillator & \pm 60 \\
\hline Non-uniformity of the $\mathrm{SiO}_{2}$ powder & \pm 30 \\
\hline \hline Total & -220 and +230 \\
\hline
\end{tabular}

Table 1 Summary of the systematic errors 


\section{Figure captions}

[Fig.1] The experimental setup in this experiment. Circular inset: a magnified view of the source region.

[Fig.2] (a) The energy spectrum of the photon emitted from the o-Ps at time window of 160-710 ns. The solid line, almost indistinguishable from the data below $508 \mathrm{keV}$, shows the simulated spectrum of the $3 \gamma$-decay. (b) The enlarged view of the peak at $511 \mathrm{keV}$ after subtracting the $3 \gamma$ contribution from the o-Ps spectrum. The solid curve shows the fitting result. (c) The pickoff annihilation rate as a function of time. The solid curve shows the fitting results, and the $\lambda_{\text {pickof } f} / \lambda_{0}$ at $t=\infty$ is presented with the dashed line.

[Fig.3] The time spectrum of the CsI scintillators at the photon-energy window of $300-410 \mathrm{keV}$. The dashed curve shows the fitted decay curve.

[Fig.4] The history of the measurements of o-Ps decay rate. The QED prediction includes the $O\left(\alpha^{2}\right)$ corrections. 


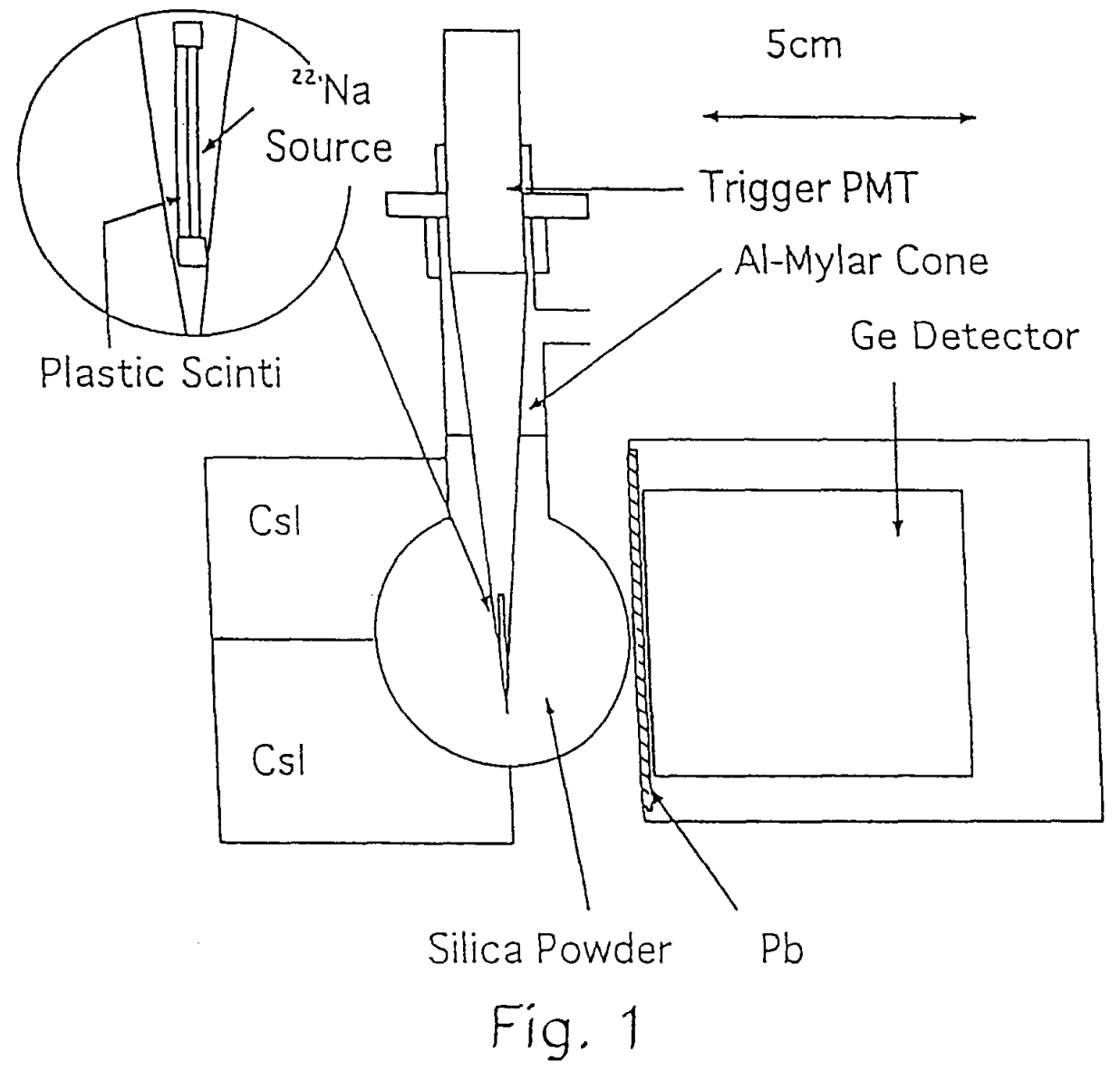



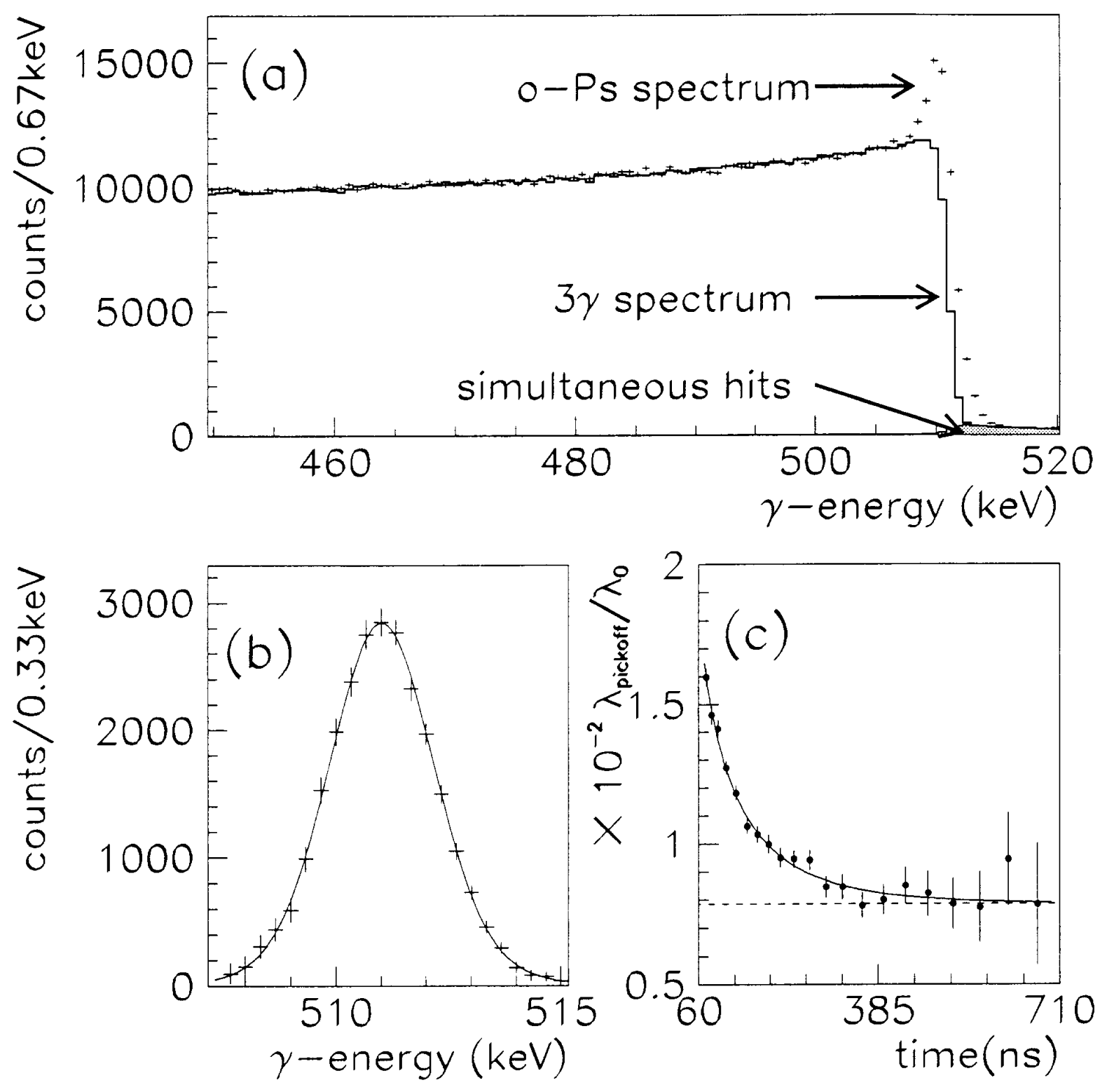

Fig. 2 


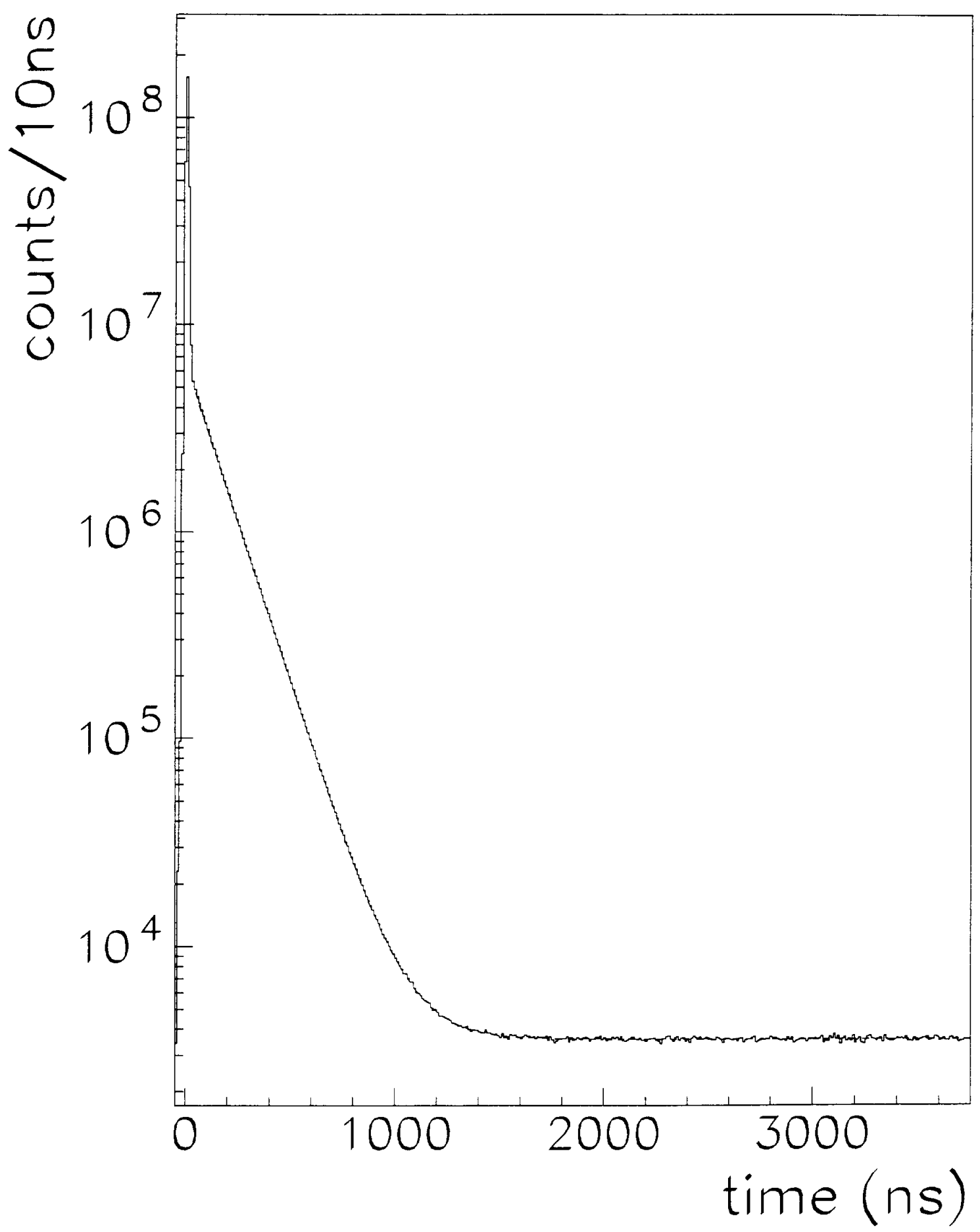

Fig. 3 


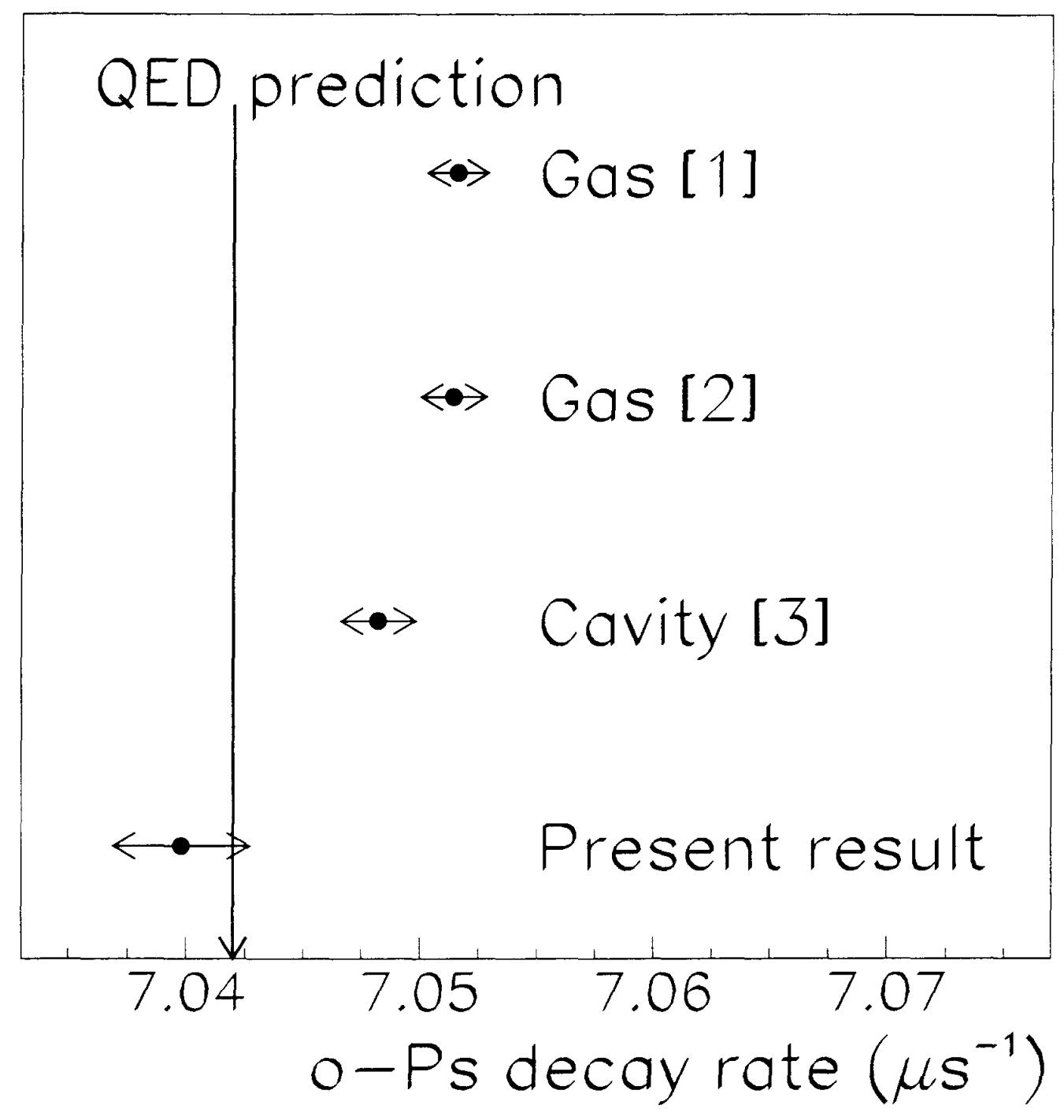

Fig. 4 
